\title{
Plattformbasiertes Sharing und Pooling im Verkehrssektor - ein Systematisierungsansatz
}

\author{
Im Verkehrssektor manifestiert sich die Digitalisierung zunehmend durch plattformbasierte \\ Geschäftsmodelle, die Mobilitätsdienstleistungen vermitteln. Auf diese Weise entsteht \\ die Möglichkeit, Fahrten und Fahrzeuge mit anderen, meist fremden Mitreisenden zu \\ teilen. Durch Suchfunktionen und Matchmaking können attraktive Angebote mit hoher \\ Dienstleistungsqualität „on demand“ aufgebaut werden, die eine stärkere Individualisierung \\ der Mobilität als der noch weitgehend starre Nahverkehr ermöglichen.
}

Der Begriff der Digitalisierung im erweiterten Sinne wird als eine momentan ablaufende Transformation der Gesellschaft und der Wirtschaft verstanden. ${ }^{1}$ Der digitale Wandel beruht auf der Digitalisierung von Wertschöpfungsketten, der Entstehung neuer Märkte und im Aufkommen plattformbasierter Geschäftsmodelle. ${ }^{2}$ Letztere verknüpfen Anbieter und Nachfrager mit Hilfe digitaler Lösungen. Durch Digitalisierung werden Transaktionskosten abgebaut und Mass Customisation ermöglicht, d.h. eine individuelle Produktgestaltung und Kundenansprache bei gleichzeitig hohen Umsätzen. Durch den Abbau von Transaktionskosten können Netzwerkeffekte bisher ungeahnter Größe realisiert werden: Der Wert der Plattform für Anbieter und Nachfrager steigt mit der Größe ihres Umsatzes.

Zwar hat die Digitalisierung schon seit längerem in die Verkehrsbranche Einzug gehalten, z. B. in Form digitaler

(C) Der/die Autor(en) 2020. Open Access: Dieser Artikel wird unter der Creative Commons Namensnennung 4.0 International Lizenz (http:// creativecommons.org/licenses/by/4.0/deed.de) veröffentlicht.

Open Access wird durch die ZBW - Leibniz-Informationszentrum Wirtschaft gefördert.

* Die Autoren bedanken sich für wertvolle Hinweise, die sie zu dem in diesem Beitrag angewendeten Ansatz auf der Konferenz „Verkehrsökonomik und -politik" an der Technischen Universität Berlin im Mai 2019 erhalten haben.

1 R. Frisch: Drei Megatrends prägen den Nahverkehr, Interview, in: Der Nahverkehr, Nr. 11, 2017, S. 18-19; A. Wittmer, E. Linden: Zukunft Mobilität: Gigatrend Digitalisierung, Universität St. Gallen, 13.1.2018, https://www.alexandria.unisg.ch/253291/ (1.8.2019); A. Heß, S. Polst: Mobilität und Digitalisierung: Vier Zukunftsszenarien, Bertelsmann Stiftung, 2017, https://www.bertelsmann-stiftung.de/fileadmin/files/ BSt/Publikationen/GrauePublikationen/Studie LK Mobilitaet-undDigitalisierung__Vier-Zukunftsszenarien_2017.pdf (1.8.2019).

2 K. Lütjens et al.: Innovationen im Zuge der Digitalisierung des Personenverkehrs, in: Wirtschaftsdienst, 98. Jg. (2018), H. 7, S. 512-518, https://www.wirtschaftsdienst.eu/inhalt/jahr/2018/heft/7/beitrag/innovationen-im-zuge-der-digitalisierung-des-personenverkehrs.html (7.1.2020).
Vertriebswege oder Echtzeitreiseinformationen während der Reise. Allerdings blieben disruptive Verschiebungen aufgrund neuer Geschäftsmodelle in der digitalen Welt (digitale Transformation) bisher aus. Im Verkehrssektor sind die plattformbasierten Modelle sehr eng mit der Möglichkeit verbunden, Fahrten und Fahrzeuge zu teilen. Wie mögliche Entwicklungen aussehen können, zeigt die Fahrtenvermittlungsplattform Uber. ${ }^{3}$ Dieser Effekt wird zuweilen auch „Uberisation“ genannt.. Neben reiner Fahrtvermittlung bietet Uber inzwischen auch eine Funktion gepoolter Fahrten an, und hier vermischt sich das Geschäftsmodell mit früheren Angeboten aus dem öffentlichen Personennahverkehr (ÖPNV) - des Anrufsammeltaxis. In der Tat finden sich in der wissenschaftlichen Literatur Abgrenzungen der Begriffe Sharing und Pooling. Diese Abgrenzungen beschreiben die Konzepte

3 M. Iqbal: Uber Revenue and Usage Statistics, Business of Apps, 10.5.2019, https://www.businessofapps.com/data/uber-statistics/ (1.8.2019).

4 D. A. Hensher: Future bus transport contracts under a mobility as a service (MaaS) regime in the digital age: Are they likely to change?, in: Transportation Research Part A: Policy and Practice, 98. Jg. (2017), S. 86-96, https://ses.library.usyd.edu.au/bitstream/2123/17497/1/ Thredbo_15_Thredbo_15_Paper_2.pdf (7.1.2020).

Kathrin Viergutz, M. Sc., Dr. Sven Maertens, Benedikt Scheier, M. Sc., Klaus Lütjens, Dipl.Volkswirt, Mirko Goletz, Dipl.-Volkswirt, Wolfgang Grimme, Dipl.-Kfm., und Prof. Dr. Gernot Liedtke sind wissenschaftliche Mitarbeiter im Bereich Verkehrsökonomie am Deutschen Zentrum für Luft- und Raumfahrt (DLR). 
allgemein und greifen damit für die Einordnung aktuell entstehender Mobilitätskonzepte, bei denen sich durch neue Geschäftsmodelle Mischformen mit völlig neuen Nutzungszwecken und Anwendungsmöglichkeiten ergeben, oft zu kurz.

\section{Sharing, Pooling und Plattformen im digitalen Wandel}

Bei Fragen der Marktpotenziale, Effizienzgewinne und Regulierungsnotwendigkeit neuer Geschäftsmodelle spielt eine grundsätzliche Rolle, worin die Funktionalität und der Mehrwert einer neuen Mobilitätsplattform zum „Poolen“, „Renten“ und „Sharen“ von Fahrten und Fahrzeugen bestehen.

\section{Literaturbasierte Begriffs- und Konzeptabgrenzung}

Haucap stellt heraus, dass „das Teilen von Ressourcen [...] nichts grundlegend Neues" ${ }^{5}$ ist. Als Beispiele nennt er Lesezirkel und die gemeinsame Nutzung von Landund Forstmaschinen mithilfe von Maschinenringen. Die Digitalisierung erweitert die Möglichkeiten des Teilens. Constantiou betont, dass Sharing-Konzepte skalierbar sind, sofern sich eine kritische Masse an Nutzern findet, die mithilfe digitaler Plattformen organisiert wird. ${ }^{6}$ Die Plattformen nutzen Algorithmen sowie nutzergenerierte Daten und nehmen die Rollen von Vermittlern ein. Laione beschreibt Sharing als eine Möglichkeit des Zugangs zu Gütern und Dienstleistungen. ${ }^{7}$ Filippas et al. stellen fest, dass Sharing-Konzepte es Eigentümern von Gebrauchsgütern ermöglichen, diese an Dritte zu vermieten und so die Nutzung zu erhöhen. ${ }^{8}$

Das Konzept des Poolings hingegen charakterisiert Laione durch einen Peer-to-Peer-Ansatz, der aus Kunden eine Gemeinschaft (Community) macht. Eine ähnliche Abgrenzung findet auch Constantiou: Sharing Economy sei geprägt durch die sozioökonomischen Trends des zugangsbasierten Konsums, der durch Vertrauen und Kundenbewertungen determinierten Peer-to-Peer-Transaktionen sowie der Allokation nicht-genutzer Ressourcen. ${ }^{9}$

5 J. Haucap: Ökonomie des Teilens - nachhaltig und innovativ? Die Chancen der Sharing Economy und ihre möglichen Risiken und Nebenwirkungen, DICE Ordnungspolitische Perspektiven, Nr. 69, 2015, https://www.econstor.eu/bitstream/10419/106708/1/816993181.pdf (7.1.2020).

6 I. Constantiou: Digitalization \& New Dimensions of Competition: Sharing Economy Platforms, o.J.

7 C. laione: Poolism: Sharing economy vs. pooling economy, 2015, http://labgov.city/commonspress/poolism/ (7.1.2020).

8 A. Filippas, J. Horton, R. Zeckhauser: Owning, Using and Renting: Some Simple Economics of the „Sharing Economy“, 2019.

9 I. Constantiou, a. a. O.
Auch im Verkehrskontext existieren die Konzepte Pooling und Sharing, wobei die Konzepte und Definitionen nicht immer trennscharf sind. Wird das Sharing als Nutzung eines Gutes durch mehrere Personen nacheinander verstanden, die nicht Eigentümer sind, so kann auch das Geschäftsmodell klassischer Selbstfahrermietwagenfirmen als Sharing-Konzept eingeordnet werden. Dies ist jedoch durch den Begriff des Rentings (Mieten) bereits bezeichnet. Mehlert und Schiefelbusch beschreiben Ridesharing als „nicht-kommerzielle Mitnahme weiterer Personen auf einer Fahrt, die ohnehin stattfinden würde"10. Auch Meyer vertritt die Auffassung, dass unter Sharing die Mitnahme von zusätzlichen Passagieren auf einer ohnehin stattfindenden Fahrt zu verstehen ist, um existierenden Platz besser auszunutzen. ${ }^{11}$ Grundsätzlich sei Sharing im Verkehr nicht kommerziell orientiert, sondern diene eher dem Ziel einer anteiligen Kostenbeteiligung bzw. -entschädigung. Pooling charakterisiert Meyer als die geplante Zusammenlegung des Verkehrsbedarfs einzelner Personen zu einem Pool, der das Fahrzeug anmietet, um gebündelt sein Ziel zu erreichen.

In diesem Artikel wird Sharing als die Nutzung von Gütern durch unterschiedliche Personen zeitlich nacheinander bzw. im Falle unbegrenzter Ressourcen auch zeitgleich verstanden. Entscheidendes Merkmal ist, dass verschiedene Nachfrager dasselbe Gut in Anspruch nehmen, ohne sich dabei zwingend zu begegnen. Pooling hingegen bezeichnet die zeitgleiche Nutzung von Gütern durch mehrere Personen, die sich dabei begegnen. Renting wird in diesem Artikel als eine Ausprägung des Sharings verstanden

\section{Mehrwerte plattformbasierter Angebotsformen}

Plattformanbieter versuchen, Anbieter und Kunden durch Netzwerkeffekte an sich zu binden. Im Extremfall wird eine Plattform zum Monopolisten, der seine Marktmacht zur Erzielung sehr hoher Gewinne ausspielen und zu einer Wohlfahrtsreduktion führen kann. Dies kann Forderungen nach einer Regulierung oder Marktbeeinflussung nach sich ziehen, um die Wohlfahrt zu steigern. Eine zu frühe Regulierung birgt jedoch die Gefahr, dass das Entstehen neuer Geschäftsmodelle, Angebotsfunktionen und damit von Innovation und Kundennutzen verhindert wird. Mögliche Mehrwerte plattformbasierter Sharing- und Poolingangebote im Vergleich zu heutigen Markstrukturen und Funktionen sind daher zu analysieren:

10 C. Mehlert, J. Schiefelbusch: Mobility on-demand: Disruption oder Hype? Entwicklung und Zukunft von Rufbus, Sharing und Robotaxi, Der Nahverkehr, Nr. 7 und 8, 2017.

11 J. Meyer: Innovative Ride Sharing Concepts, ITB Kongress, 2019. 
Tabelle 1

Matrix zur Abgrenzung unterschiedlicher Nutzungs- und Plattformkonzepte

\begin{tabular}{|c|c|c|c|c|}
\hline \multicolumn{2}{|c|}{ Nutzungskonzept } & \multirow{2}{*}{$\begin{array}{l}\text { Kein Sharing/Pooling } \\
\text { Veränderung der Eigentums- bzw. } \\
\text { langfristigen Besitzverhältnisse }\end{array}$} & \multirow{2}{*}{$\begin{array}{l}\text { Sharing } \\
\text { Wechselnder Besitz/Nutzung im } \\
\text { Zeitablauf; (bei begrenzten/einmaligen } \\
\text { Ressourcen: Nutzung nacheinander) }\end{array}$} & \multirow{2}{*}{$\begin{array}{l}\text { Pooling } \\
\text { Nutzung durch mehrere Personen } \\
\text { gleichzeitig (Bündelung) }\end{array}$} \\
\hline & & & & \\
\hline \multirow[t]{2}{*}{$\begin{array}{l}\text { Keine } \\
\text { Plattform }\end{array}$} & offline & $\begin{array}{l}1 \text { Konsumgüterverkauf durch stationären } \\
\text { Handel; Essenslieferservice über Flyer }\end{array}$ & $\begin{array}{l}5 \text { Milchpumpenverleih einer Apotheke; } \\
\text { lokale Dienstleistungen }\end{array}$ & $\begin{array}{l}9 \text { Bibliotheksarbeitsplatz; Bürogemein- } \\
\text { schaft; Hostel (geteiltes Zimmer); } \\
\text { Fitnessstudio, -kurs; Kino }\end{array}$ \\
\hline & online & $\begin{array}{l}2 \text { Essenslieferservice; Onlinehändler (z. B. } \\
\text { Tchibo.de; Amazon als Händler) }\end{array}$ & $\begin{array}{l}6 \text { Onlinebasierter Verleih ( z. B. Spielzeug, } \\
\text { Kostüme); Hotel(kette) mit Webvertrieb }\end{array}$ & $\begin{array}{l}10 \text { Hostel (geteiltes Zimmer) mit Web- } \\
\text { vertrieb }\end{array}$ \\
\hline \multirow[t]{2}{*}{ Plattform } & offline & $\begin{array}{l}3 \text { Einkaufszentrum; Shop-Angebot an } \\
\text { Verkehrsknoten; Flohmarkt; Schwarzes } \\
\text { Brett zum An- und Verkauf }\end{array}$ & 7 Speed Dating & $\begin{array}{l}11 \text { Schwarzes Brett für Mitwohnmög- } \\
\text { lichkeiten }\end{array}$ \\
\hline & online & $\begin{array}{l}4 \text { Online-Marktplatz mit unterschiedli- } \\
\text { chen Händlern (z. B. Amazon Market- } \\
\text { place, eBay); Onlineauktionen (z. B. } \\
\text { eBay); App für verschiedene Essenslie- } \\
\text { ferservices (z. B. Lieferando) }\end{array}$ & $\begin{array}{l}8 \text { Unterkunftsplattformen (z. B. Airbnb, } \\
\text { booking.com, fewo-direkt, trivago, } \\
\text { hotels.com); Streaming-Dienste (z.B. } \\
\text { Deezer, Netflix, Youtube) }\end{array}$ & $\begin{array}{l}12 \text { Mitwohnzentrale (z. B. Vermietung } \\
\text { eines Betts/Zimmers bei Airbnb, } \\
\text { Couchsurfing.com) }\end{array}$ \\
\hline
\end{tabular}

Quelle: eigene Darstellung.

- Netzwerkexternalitäten: Mit der Zahl der teilnehmenden Anbieter und Nachfrager steigt aufgrund der inhaltlichen Diversität der Angebote die Wahrscheinlichkeit zu höherer Passgenauigkeit.

- Qualitätssicherung: Ratingfunktionen der Plattformen spornen Dienstleister an, eine hochwertige Dienstleistungsqualität zu erbringen, und reduzieren Risiken und Hemmungen auf Kundenseite. Manche Plattformen geben Kunden zudem Sicherheit, indem sie bei Ausfall eines Dienstleisters oder einer schlechten Dienstleistungsqualität Haftung übernehmen. Bei einer zu groBen Marktmacht der Plattform gegenüber Dienstleistern kann dies aber auch zu einem Machtmissbrauch führen.

- Suchkostenreduktion: Durch höheren Auswahlnutzen besteht die Gefahr, dass Kunden kognitiv nicht mehr in der Lage sind, sich für einen bestimmten Anbieter zu entscheiden. An dieser Stelle helfen viele Plattformen in Form von Filtern und Vorschlagsfunktionen bei der Entscheidungsfindung.

- Fixkostendegression: Die Kosten der Entwicklung und des Betriebs einer Plattform pro Transaktion sinken, je größer der Umsatz einer Plattform ist.

- Mehrwertdienste: Plattformen können nicht nur zwischen Anbietern und deren Kunden vermitteln, sondern den Kunden zusätzliche komplementäre Dienstleistungen vermitteln, z. B. den Abschluss einer Versicherung. Viele Plattformen integrieren zudem analytische Funktionen für Anbieter und Kunden, angefangen von Verkaufsberichten bei eBay bis hin zu spezifischen Tools etwa für Anbieter von Verkehrsdienstleistungen, wie beispielsweise Tourenplanung, Verkehrsinformation oder Ankunftszeitprognose. Hinzu kommen digitale Funktionen zur Abrechnung bzw. Bezahlung.

Bei der Analyse eines Geschäftsmodells hinsichtlich einer Ex-ante-Regulierung kommt es also sehr darauf an, wie stark diese Elemente ausgeprägt sind. Solange verschiedene Plattformen mit unterschiedlichen Konzepten aktiv sind, ist noch ein ausgeprägter Innovationswettbewerb festzustellen, bei dem ein frühzeitiges Regulieren und/ oder Standardisieren schädlich sein kann. Die Netzwerkeffekte und niedrigen Transaktionskosten digitaler Plattformen eignen sich insbesondere auch für neue Ansätze, Anbieter und Nachfrager selbst kurzfristig zusammenzubringen, wie etwa durch Sharing und Pooling.

\section{Abgrenzung verschiedener Nutzungs- und Plattformkonzepte}

Tabelle 1 stellt den Bezugsrahmen zur Einordnung von Angeboten nach Nutzungskonzept (spaltenweise) sowie Grad der Bündelung von Anbietern auf Plattformen und Grad der Digitalisierung (zeilenweise) dar. Im Zeitablauf ist in vielen Fällen eine Verschiebung von der analogen in die digitale Welt und/oder hin zu Plattformen zu beobachten, teils auch bei Erhöhung des Konzentrationsgrades:

- Essensbestellungen werden zunehmend nicht mehr flyerbasiert und telefonisch (Feld 1) oder direkt über die Webpräsenz des Lieferdienstes (Feld 2), sondern über 
App-basierte Plattformen aufgegeben, bei denen eine zunehmende Marktkonzentration zu entstehen scheint (Feld 4).

- Medien (Musik, Film, Bücher) wurden zunächst als physische Produkte zunehmend über das Internet vertrieben (von Feld 1 in Feld 2/4), werden aufgrund ihrer Digitalisierbarkeit aber mittlerweile zunehmend durch Sharing-Dienste angeboten (Feld 8).

- Klassische Sharing-Angebote, wie etwa Hotelzimmer, werden zunehmend über Plattformen digital vermarktet (von Feld 5/6 in Feld 8). Um die Marktmacht der Plattformen einzudämmen, versuchen Hotels und Hotelketten immer häufiger, Kunden über besondere Anreize wie Gratisleistungen zu einer Direktbuchung zu bewegen.

Aufgrund der beschriebenen Netzwerkeffekte und niedrigen Transaktionskosten hat sich das Spektrum des Poolings stark vergrößert. Insbesondere bei knappen Gütern sowie Unikaten (etwa Gebrauchtartikelhandel) besteht der Trend hin zu monopolistischen Plattformen. Bei über Kapazitätsmanagementsysteme in Echtzeit gesteuerten Angeboten wie Hotelzimmern sind auch parallel mehrere Buchungsplattformen möglich und eine Monopolisierung des Vertriebs ist noch nicht absehbar.

\section{Sharing- und Pooling-Angebote im Verkehrssektor}

Innovative Mobilitätskonzepte bieten das Potenzial, sowohl angebots- wie auch nachfrageseitig stark am Markt orientiert gestaltet zu werden. Dies setzt für Anbieter ein Verständnis des Marktes und dessen zukünftiger Entwicklung voraus. ${ }^{12}$ Tabelle 2 ergänzt Tabelle 1 um die Einordnung von Transportangeboten.

\section{Einordnung von klassischen Verkehrsangeboten}

Seit der Verfügbarkeit erster Fahrzeuge für den Individualtransport wie Fahrräder oder den Pkw sind der Kauf und die individuelle Nutzung das vorherrschende Nutzungskonzept. Beim etablierten Pkw-Leasing (Feld 1) handelt es sich ebenfalls um ein längerfristiges Nutzungskonzept, das sich in einigen Rechten und Pflichten und hinsichtlich der Finanzierung vom Kauf unterscheidet. Online- und plattformbasierte Varianten dieser Nutzungskonzepte existieren schon seit langem in Form herstellerübergreifender (Gebrauchtwagen-)Händler und dem Internet als weiterem Vertriebskanal (Feld 4).

12 M. Enoch, S. Potter, G. Parkhurst, M. Smith: Why do demand responsive transport systems fail?, Paper for $85^{\text {th }}$ Annual Meeting of the TRB, 2006.
Auch Sharing (bzw. Renting) und Pooling existieren im Verkehr schon lange. In der Zeit vor dem Internet: das telefonisch oder an der Straße gerufene Taxi (Sharing) (Feld 7) oder die Anmietung eines Mietwagens oder Fahrrades an entsprechenden Stationen (Renting) (Feld 5). Das prominenteste Beispiel für Pooling dürften öffentliche Verkehrsmittel im Linien- oder Charter-Verkehr (Felder 9 und 10) sein, die als Massentransportmittel Passagiere zu geringen Kosten, umweltschonend und bei geringem Raumbedarf transportieren. Aber auch klassische Mitfahrgelegenheiten fallen hierunter. Für diese Formen des Poolings gab es bereits vor dem Durchbruch des Internets Plattformangebote wie Reisebüros oder Schwarze Bretter bzw. die klassischen Computerreservierungssysteme als Schnittstellen zwischen Anbietern und Vertrieb (Feld 11).

Die Verbreitung des Internets um die Jahrtausendwende ermöglichte für Anbieter von Sharing-, Renting- und Pooling-Konzepten zunächst die Nutzung eines neuen Vertriebskanals, z.B. die Direktvermarktung von Flugtickets über die eigene Webseite (Feld 10), wovon insbesondere die Billigfluggesellschaften frühzeitig Gebrauch machten. Umgekehrt bedeutete dies für die Kunden den direkten Zugang zum jeweiligen Anbieter, ohne ein Reisebüro nutzen zu müssen. Auch Mitfahrzentralen (Feld 12) haben über Netzwerkeffekte frühzeitig vom Internet profitiert, weil bei geringen technischen Anforderungen an entsprechende Webseiten deren Fähigkeit zur Koordinierung von Fahrtanbietern und Mitfahrern massiv gestiegen ist.

Nutzungskonzepte im Verkehr durch neue Technologien

Die nachfolgend beschriebenen Verkehrsangebote haben sich überwiegend in den letzten Jahren entwickelt. Allen Angeboten ist gemein, dass sie hohe Anforderungen an die technische Ausrüstung von Anbieter, Fahrzeug, Nutzer und umgebender Infrastruktur stellen, sodass die Angebote erst in jüngster Zeit realisiert werden konnten. Darüber hinaus ist die durchgehende Digitalisierung und darauf aufbauend ein hoher Automatisierungsgrad aller Abläufe erforderlich, damit diese Verkehrsangebote in Echtzeit und zu wettbewerbsfähigen Kosten, z.B. auch bei Anmietung von Fahrzeugen für wenige Minuten, darstellbar sind.

1. Nicht-plattformbasiertes Fahrzeug-Sharing (Feld 6). Auf dem deutschen Markt war die Firma Stattauto (heute Greenwheels) 1988 der Pionier für dieses Nutzungskonzept mit Pkw, allerdings mit festen Stationen für die Fahrzeuge. Flinkster als Tochter der Deutschen Bahn weist mit 4000 Fahrzeugen in 400 Städten die meisten Fahrzeuge und die größte überregionale Flächenabdeckung in Deutschland auf. Streng genommen handelt es sich bei Flinkster sogar um ein Platt- 
Tabelle 2

Transportangebote nach Nutzungskonzept, Bündelung von Anbietern und Offline/Online-Umsetzung

\begin{tabular}{|c|c|c|c|c|}
\hline \multicolumn{2}{|c|}{ Nutzungskonzept } & \multirow{2}{*}{$\begin{array}{l}\text { Ownership/Leasing } \\
\text { Längerfristiges, ausschließ- } \\
\text { liches Eigentum (Kauf) oder } \\
\text { Besitz (Leasing) }\end{array}$} & \multirow{2}{*}{$\begin{array}{l}\text { Sharing/Renting } \\
\text { Sequenzieller, ausschließlicher Besitz; } \\
\text { bei begrenzten (einmaligen) Ressourcen: Nut- } \\
\text { zung durch mehrere Personen nacheinander }\end{array}$} & \multirow{2}{*}{$\begin{array}{l}\text { Pooling } \\
\text { Sequenzieller, gemeinsamer Besitz } \\
\text { (Nutzung durch mehrere Personen } \\
\text { gleichzeitig, Bündelung von Passagier- } \\
\text { strömen) }\end{array}$} \\
\hline & & & & \\
\hline \multirow[t]{2}{*}{$\begin{array}{l}\text { Keine } \\
\text { Plattform }\end{array}$} & offline & $\begin{array}{l}1 \text { Kauf/Leasing von Fahrzeu- } \\
\text { gen beim Hersteller oder } \\
\text { Vertragshändler und eigene } \\
\text { Nutzung }\end{array}$ & $\begin{array}{l}5 \text { Klassisches Taxiunternehmen; Selbstfahrer- } \\
\text { Mietwagen (z. B. Hertz, Sixt); Fahrradverleih am } \\
\text { Strand }\end{array}$ & $\begin{array}{l}9 \text { Linien- und Charterverkehr (Bus, Bahn, } \\
\text { Flug), beim Anbieter offline gebucht; } \\
\text { traditionelle Mitfahrgelegenheit mit } \\
\text { persönlicher Absprache }\end{array}$ \\
\hline & online & $\begin{array}{l}2 \text { Kauf/Leasing von Fahr- } \\
\text { zeugen auf Webseite des } \\
\text { Herstellers oder Händlers } \\
\text { und eigene Nutzung }\end{array}$ & $\begin{array}{l}6 \text { Selbstfahrer-Mietwagen beim Anbieter online } \\
\text { gebucht; App-basiertes Fahrzeugsharing: Autos } \\
\text { stationsgebunden: Flinkster, Greenwheels, } \\
\text { Ubeeqo; Autos Free Float: Share Now (Fusion } \\
\text { von Car2go und Drive Now), Cambio, Oply, } \\
\text { Miles; Fahrräder: StadtRAD, Nextbike; E-Roller: } \\
\text { Emmy; E-Scooter: Lime, Circ, Tier, Voi }\end{array}$ & $\begin{array}{l}10 \text { Linien- und Charterverkehr (Bus, } \\
\text { Bahn, Flug) beim Anbieter direkt } \\
\text { online gebucht; Demand-Responsive- } \\
\text { Transport-Betreiber (DRT-Betrieber) } \\
\text { mit angestellten Fahrern, z. B. Moia, } \\
\text { Clevershuttle }\end{array}$ \\
\hline \multirow[t]{3}{*}{ Plattform } & offline & $\begin{array}{l}3 \text { Kauf/Leasing von Fahrzeu- } \\
\text { gen bei herstellerübergrei- } \\
\text { fendem Händler und eigene } \\
\text { Nutzung }\end{array}$ & $\begin{array}{l}7 \text { Telefonbasierte Taxizentralen für klassische } \\
\text { Taxiangebote; Mitfahrerbänke }\end{array}$ & $\begin{array}{l}11 \text { Schwarzes Brett für Mitfahrgelegen- } \\
\text { heiten; Linienverkehr (Bus, Bahn, Flug) } \\
\text { beim Reisebüro offline gebucht }\end{array}$ \\
\hline & online & $\begin{array}{l}4 \text { Kauf/Leasing von Fahrzeu- } \\
\text { gen auf herstellerunabhän- } \\
\text { giger Webseite (etwa über } \\
\text { AutoScout24) und eigene } \\
\text { Nutzung }\end{array}$ & $\begin{array}{l}8 \text { Fahrdienstvermittlungen: Free Now (früher } \\
\text { myTaxi), UberX, UberBlack, UberPop (private } \\
\text { Fahrer); App-basiertes Fahrzeug-Sharing mit } \\
\text { Einbindung fremder Fahrzeuge: Flinkster mit } \\
\text { Partnernetzwerk; private und gewerbliche Fahr- } \\
\text { zeuge: Drivy, SnappCar }\end{array}$ & $\begin{array}{l}12 \text { Online-Plattform für Mitfahrgelegen- } \\
\text { heiten, z. B. Mitfahrzentrale.de, Bla- } \\
\text { BlaCar; vergleichende Reiseplanung } \\
\text { und Buchung von Bus, Bahn, Flug } \\
\text { und Mitfahrgelegenheiten, z. B. Qixxit, } \\
\text { fromAtoB, Omio }\end{array}$ \\
\hline & online & & \multicolumn{2}{|c|}{$\begin{array}{l}13 \text { Kombiniertes Sharing und Pooling mit eigenen und fremden Transportangeboten, z. B. Ver- } \\
\text { kehrsverbünde (z. B. HVVISwitch in Hamburg), DB-Navigator, Sixt, Reach Now (früher Moovel) }\end{array}$} \\
\hline
\end{tabular}

Anmerkung: In den 2010er Jahren entwickelte Angebote sind in kursiver Schrift dargestellt.

Quelle: eigene Darstellung.

formmodell, da auch auf Fahrzeuge von 20 kleineren lokalen Carsharing-Partnern zugegriffen werden kann. Das heute in Großstädten zunehmend verbreitete FreeFloating-Modell, bei dem Fahrzeuge an beliebigen Orten innerhalb eines festgelegten Geschäftsgebietes anmiet- und abstellbar sind, wurde mit der Gründung von Car2Go 2009 in Deutschland eingeführt. Mit diesem Modell folgte 2015 das E-Roller-Sharing mit Jaano (Betriebseinstellung 2017) und Emmy. Auf Basis der Elektrokleinstfahrzeuge-Verordnung (eKFV) vom Juni 2019 werden nun auch E-Scooter im Rahmen des FreeFloating-Sharing-Modells angeboten. Die wesentlichen technischen Anforderungen für diese Geschäftsmodelle sind die jederzeitige Ortbarkeit der Fahrzeuge, die Inbetriebnahme per Kundenkarte oder App sowie die elektronische Buchung und Abrechnung. Anbieter konkurrieren über die Art der Fahrzeuge, die Bedienfreundlichkeit der App, den Preis und unterschiedliche Tarifmodelle. Während die Anmeldung oft kostenlos ist, wird die Nutzung häufig pro Minute oder Kilometer und teilweise zusätzlich pro Anmietung berechnet. Ergänzt wird dies für längere Nutzungen durch Pakete aus Zeitraum und Kilometerzahl (bei Car2Go z.B. 24 Stunden,
200 Kilometer). Fixe Monatsgebühren oder PrepaidZahlungen werden in der Regel nicht erhoben oder sind wie bei den Anbietern Cambio und Ubeeqo mit einer Verringerung der Kosten pro Nutzung verbunden (zweiteiliger Tarif). Aufgrund der möglichen Online-Buchung klassischer Mietwagen und der mehrtätigen Nutzung von Carsharing-Fahrzeugen verschwimmt die Grenze zwischen beiden Konzepten, sodass von einigen Anbietern bereits beide Optionen in einer App kombiniert angeboten werden.

2. Nicht-Plattformbasiertes Pooling: Demand-Responsive-Transport-Fahrdienste (DRT-Fahrdienste) (Feld 10). Nachfrageorientierte Verkehrsangebote, die nur bei einer temporären direkten Nachfrage durch Fahrgäste verkehren, existieren weltweit bereits seit vielen Jahrzehnten. Besonders in Ländern des "globalen Südens“ spielen diese häufig auch als para-transit bezeichneten Verkehre eine wichtige Rolle. Gemeint sind gemeinhin auf fixen Routen, aber ohne feste Fahrpläne verkehrende Linien eines de facto öffentlichen Personennahverkehrs, der durch private, individualwirtschaftlich agierende Akteure meist mit Kleinbussen betrieben 
wird. ${ }^{13}$ Auch in den USA finden sich unter dem Begriff Van-Pool-Services ähnliche Angebote, die einen täglichen Service anbieten, der sich meist an Pendler richtet. ${ }^{14}$ Vor diesen Angeboten hat auch die Digitalisierung keinen Halt gemacht, sowohl in Ländern des "globalen Südens“ als auch des "globalen Nordens“ können verstärkt gepoolte Fahrten mit Minibussen durch Kunden bestellt werden. Diese digitalisierten Angebote werden als Micro-Transit bezeichnet. Angebote dieser Art können in zwei Dimensionen flexibel sein: Hinsichtlich der Routenwahl und/oder der Abfahrtszeiten, wodurch sich unterschiedliche Implikationen ergeben. ${ }^{15}$

In Mexiko Stadt bietet beispielsweise der Anbieter Jetty ${ }^{16}$ ein solches Angebot an; der kalifornische Anbieter Chariot war längere Zeit in San Francisco aktiv, musste Anfang 2019 sein Angebot jedoch wieder einstellen. In Deutschland entstehen seit wenigen Jahren vermehrt flexible Angebote, die Mini-Busse zur Angebotserstellung nutzen: Moia und Clevershuttle in mehreren Städten Deutschlands oder der in Berlin verkehrende BerlKönig, der durch das städtische Verkehrsunternehmen Berliner Verkehrsbetriebe (BVG) mitbetrieben wird. Charakteristisch für Micro-Transit ist, dass das Angebot durch einen zentralen Anbieter bereitgestellt wird, der sowohl für die Kundenschnittstelle (meist eine App) als auch für das Backend und die Erstellung des Angebots (Fuhrparkmanagement und Fahrer) zuständig ist. Damit lässt sich dieser Angebotstyp von den plattformbasierten Sharing-Modellen abgrenzen.

3. Plattformbasiertes Sharing: Taxi-/Fahrdienstvermittlungen und Fahrzeug-Sharing mit Partnern (Feld 8). Plattformbasierte Services bündeln Transportangebote verschiedener Anbieter in einer App. Im Falle plattformbasierter Fahrdienstvermittlungen betrifft dies zum einen die Bündelung professioneller (UberX, UberBlack, myTaxi) oder privater Fahrdienstleister (UberPop). Zur Nutzung des Dienstes benötigen sowohl der Kunde als auch der Fahrer ein Smartphone oder Tablet sowie die App des Plattform-Anbieters. Die technische Plattform an sich und die zugehörigen Apps stellen neben der Plattform-Marke die wesentlichen wettbewerbsrelevanten Infrastrukturen des Plattform-Anbieters dar. Dazu gehören Funktionen wie die algorith-

13 R. Behrens, D. McCormick, D. Mfinanga (Hrsg.): Paratransit in African Cities, London 2016, https://doi.org/10.4324/9781315849515 (7.1.2020).

14 C. Musili, D. Salon: Do Private Transport Services Complement or Compete against Public Transit? Evidence from the Commuter Vans in Eastern Queens, New York, in: Urban Science, 3. Jg. (2019), H. 24.

15 S. Shaheen, N. Chan, A. Bansal, A. Cohen: Shared Mobility: Definitions, Industry Developments, and Early Understanding, White Paper, UC Berkeley, 2015.

16 Vgl. Website von Jetty, https://www.jetty.mx/en/ (23.1.2020). menbasierte Zuordnung (Matching) zwischen Fahrgast und Fahrer, die Preisgestaltung je nach Region und Fahrtstrecke sowie das Vertrauensmanagement über Standardisierungsvorgaben, Prüfung der Fahrer und Nutzerfeedback. Darüber hinaus vermitteln die Plattformen Drivy und SnappCar das Sharing privater und gewerblicher Fahrzeuge ohne Fahrer. Laut Unternehmens-Webseite werden auf Drivy in Deutschland 6000 Fahrzeuge angeboten, womit die Fahrzeugzahl fast so hoch ist wie beim Marktführer der nicht-plattformbasierten Anbieter, Share Now, mit 7400 Fahrzeugen. Allerdings dürfte die Verfügbarkeit je Fahrzeug bei Drivy deutlich geringer sein, da diese durch den Eigentümer auch privat genutzt werden. Durch die größere Sichtbarkeit der Fahrzeuge verzeichnet Share Now 2019 in Deutschland 3 Mio. Kunden im Vergleich zu 300000 bei Drivy. Auch das Carsharing-Angebot von Flinkster ist als Plattform zu nennen, da diese Fahrzeuge anderer lokaler Carsharing-Anbieter einbindet.

4. Plattformbasiertes Pooling: Vergleichende Reiseplanung (Feld 12). Hier handelt es sich um Apps zur Reiseplanung und Buchung von Bus, Bahn, Flug ohne eigene Bereitstellung von Transportdiensten. Der Fokus liegt auf dem zeitlichen und preislichen Vergleich der Verkehrsmittel auf der Hauptroute zwischen Städten mit im Flugverkehr teils weltweiter Abdeckung. Die größte Herausforderung für diese Anbieter ist das Schnittstellenmanagement mit einer Vielzahl von Transportdienstleistern.

5. Plattformbasiertes kombiniertes Sharing und Pooling (Feld 13). Unter diese Kategorie fallen Anbieter, über die Nutzer sowohl auf Sharing- als auch Pooling-Transportdienste zugreifen können. Lokal agieren Städte und Regionen mit ihren Verkehrsverbünden seit jeher in einer Plattformrolle, durch die unterschiedliche Transportanbieter für Regionalbahn, Schnellbahn, U-Bahn, Bus und Fährschiffe in einem regionalen Angebot zusammengefasst werden, das heutzutage zunehmend online verfügbar ist. Zudem können Verkehrsverbünde bei ihren Abonnenten zusätzlich das Fahrzeug-Sharing fördern, etwa in Form der Kooperation des Hamburger Verkehrsverbunds (HVV) mit Car2go, DriveNow, Cambio und dem Hamburger StadtRad unter dem Label Switchh in Ergänzung zum HVV-Abo. Neben Startguthaben und Freiminuten stellt Switchh auch Parkplätze für die Fahrzeuge dieser Anbieter bereit. Überregional positionieren sich viele Anbieter mit Plattformen mit einer nationalen, europäischen oder sogar weltweiten Abdeckung, die in der Regel verschiedene Verkehrsmittel, darunter als Kernkomponente auch eigene Sharing- und Pooling-Angebote, beinhalten. a) Die App DBNavigator der Deutschen Bahn konzentriert sich auf 
den eigenen Schienenverkehr, ermöglicht jedoch auch die Buchung von Regionalzügen anderer Anbieter, CityTicket-Verbindungen bis hinein in die lokalen Verkehrsverbünde und erste Sharing-Angebote (Call a Bike, DB Shuttle Service). b) Das Joint Venture von Daimler und BMW moovel hat sich 2019 unter dem Namen Your Now neu positioniert. Dessen App Reach Now bündelt innerhalb von zurzeit 20 Städten den ÖPNV-Verkehr, das eigene Carsharing Share Now (Car2go und Drive Now), NextBike und den Taxi-Dienst Free Now (früher myTaxi). c) Sixt positioniert sich mit seiner App SixtOne ebenfalls als Mobilitätsanbieter mit eigenen Fahrzeugen und den Funktionen Rent, Share und Ride (hier auch Nutzung von Fremdanbietern) mit weltweiter Abdeckung in einer Vielzahl von Städten. d) Apps wie Qixxit, fromAtoB und Omio verzichten komplett auf eigene Transportdienste, sondern ermöglichen in unterschiedlichem Ausmaß die intermodale Reiseplanung sowie Buchung und Bezahlung. Einer Einbindung des Luftverkehrs in die meisten dieser Angebote scheint insbesondere das Risiko verpasster Anschlüsse und der damit verbundenen Umbuchungskosten entgegenzustehen.

\section{Zusammenfassung und Ausblick}

Dieser Artikel grenzt die Konzepte des Sharings und Poolings anhand verschiedener Marktbeispiele und Entwicklungspfade aus dem Mobilitätsektor voneinander ab. Die bereits vorhandene Vielfalt von Marktteilnehmern verdeutlicht die laufende Mobilitätswende von der klassischen Nutzung von Individualverkehrsmitteln (Eigentum bzw. langfristiger Besitz) hin zu vermehrt kurzzeitigem Besitz (Sharing, Renting) oder einer zeitgleichen gemeinsamen Nutzung (Pooling). Erst die Digitalisierung von Prozessen und Plattformen ermöglicht es, Netzwerkeffekte und damit aus Nutzersicht ansprechende und für Betreiber ökonomisch tragfähige Angebote zu schaffen und kritische Massen zu erreichen.

Diese Entwicklungen sind insbesondere für raum- und verkehrspolitische Entscheidungsträger von Relevanz, um an positiven Wirkungen partizipieren und auf negative Wirkungen reagieren zu können. Der Ermittlung und Bewertung dieser Wirkungen und damit des Nutzens neuer Verkehrskonzepte durch geeignete Methoden und Modelle aus Wissenschaft und Forschung kommt daher eine entscheidende Bedeutung zu. Dies betrifft beispielsweise die folgenden Fragen:

- Kann die Nutzungs- und Angebotsflexibilität geteilter öffentlicher Verkehrssysteme so weit gesteigert werden, dass Besitz und Nutzung privater Pkw zurückgehen? Wie würde ein solcher Modal Shift die Verkehrssysteme und deren Wirkungen beeinflussen?

- Wie lassen sich die diskutierten Konzepte in bestehende (öffentliche) Verkehrssysteme integrieren?

- Können ökonomisch tragfähige sharing- oder poolingbasierte Verkehrskonzepte für den ländlichen Raum entwickelt werden?

- Tragen Netzwerkeffekte von Plattformen zu einer Monopolisierung bei und inwiefern wirkt sich dies negativ auf Nutzer und Gesellschaft aus?

- Effizienz- und Effektivitätsgewinne lassen sich nur durch von Nutzern bereitgestellte Daten erreichen. Wie lassen sich die Anforderungen an Datenschutz und Privatsphäre gewährleisten?

- Inwiefern könnte eine Automatisierung der Fahrzeuge die ökonomische Tragfähigkeit unterstützen?

Digitalisierung und die Entstehung von Plattformen hat zu neuen, netzwerkbasierten Angeboten in verschiedenen Lebens- und Wirtschaftsbereichen - mit teils disruptiven Wirkungen auf bestehende Systeme - geführt. Die genauen Wirkungen neuer Angebote und Konzepte etwa auf Nutzer, Anbieter, Angestellte und die Umwelt sind jedoch bislang weitgehend unklar. Ähnliches gilt für geeignete Regulierungsansätze. Der interdisziplinären Forschung an diesen Themen kommt daher eine entscheidende Rolle zu.

Title: Platform-based Sharing and Pooling for the Transport Sector - a Systematic Approach

Abstract: Digitisation enables platformbased business models in the transport sector, providing new types of mobility services. These platforms match supply and demand for shared or pooled transport services, with different users sharing the same vehicles in order to pool their trips. Search functions and matchmaking enhance high quality on-demand services, which allow for greater individualisation of mobility compared to the still largely rigid local transport systems. This article systemises these new mobility services and provides examples of recent market developments. Finally, questions are raised about the impact of these developments on, e.g., urban and rural traffic systems and welfare.

JEL Classification: R40, L91, 033 